\title{
Positivity of three-term recurrence sequences *
}

\author{
Lily L. Liu \\ School of Mathematical Sciences \\ Qufu Normal University \\ Qufu 273165, P. R. China \\ lliulily@yahoo.com.cn
}

Submitted: Oct 10, 2008; Accepted: Mar 24, 2010; Published: Apr 5, 2010

Mathematics Subject Classification: 11B37, 05A20

\begin{abstract}
In this paper, we give the sufficient conditions for the positivity of recurrence sequences defined by

$$
a_{n} u_{n}=b_{n} u_{n-1}-c_{n} u_{n-2}
$$

for $n \geqslant 2$, where $a_{n}, b_{n}, c_{n}$ are all nonnegative and linear in $n$. As applications, we show the positivity of many famous combinatorial sequences.
\end{abstract}

\section{Introduction}

The significance of the positivity to combinatorics stems from the fact that only the nonnegative integer can have a combinatorial interpretation. There has been an amount of research devoted to this topic in recent years (see $[1,2,5,9,10,14,15]$ for instance). The purpose of this paper is to present some sufficient conditions for the positivity of recurrence sequences.

Let $u_{0}, u_{1}, u_{2}, \ldots$ be a sequence of integer numbers. The sequence is called a (linear) recurrence sequence if it satisfies a homogeneous linear recurrence relation

$$
u_{n}=a_{1} u_{n-1}+a_{2} u_{n-2}+\cdots+a_{k} u_{n-k}
$$

for $n \geqslant k$, where $a_{1}, a_{2}, \ldots, a_{k} \in \mathbb{Z}$. The linear recurrence relation (1) defines a unique integer sequence $\left\{u_{n}\right\}_{n \geqslant 0}$ after the first $k$ initial terms $u_{0}, u_{1}, \ldots, u_{k-1}$ are given. Let $p(x)=x^{k}-a_{1} x^{k-1}-\cdots-a^{k}$ be its characteristic polynomial with discriminant $D$. Following [7], the positivity problem is stated as follows.

\footnotetext{
${ }^{*}$ Partially supported by the National Science Foundation of China under Grant No.10771027.
} 
Positivity Problem. Let a linear recurrence relation (1) be given together with the initial terms $u_{i}$ for $i=0,1, \ldots, k-1$. Is the recurrence sequence $\left\{u_{n}\right\}_{n \geqslant 0}$ nonnegative, i.e., does it hold that $u_{n} \geqslant 0$ for all $n$ ?

So far there have been some results on the positivity problem. For example, Halava et al [7] presented that the positivity problem is decidable for three-term recurrence sequences defined by

$$
u_{n}=a u_{n-1}+b u_{n-2}
$$

for $a, b \in \mathbb{Z}$. More precisely, we can conclude the following result from $[7]$ when $a b \neq 0$.

Theorem 1.1. Suppose that the sequence $\left\{u_{n}\right\}_{n \geqslant 0}$ satisfying the three-term recurrence relation (2) with the discriminant $D=a^{2}+4 b \geqslant 0$. Let $\lambda$ and $\Lambda$ be the smaller and larger characteristic roots respectively. Then the full sequence $\left\{u_{n}\right\}_{n \geqslant 0}$ is nonnegative if and only if one of the following conditions hold.

(i) $a>0, b<0$ and $u_{1} \geqslant u_{0} \lambda \geqslant 0$.

(ii) $a<0, b>0$ and $u_{1}=u_{0} \Lambda \geqslant 0$.

In this paper, we are mainly interested in the positivity problem of sequences satisfying the following more general recurrence

$$
a_{n} u_{n}=b_{n} u_{n-1}-c_{n} u_{n-2},
$$

where $a_{n}, b_{n}, c_{n}$ are all nonnegative and linear in $n$. There are many combinatorial sequences satisfying this recurrence. For example, the central Delannoy sequence $\{D(n)\}$ satisfies the recurrence

$$
n D(n)=3(2 n-1) D(n-1)-(n-1) D(n-2)
$$

with $D(0)=1, D(1)=3$ and $D(2)=14$ (see [12] for instance). However, we cannot get that the sequence $\{D(n)\}$ is nonnegative directly from the recurrence (4).

The paper is organized as follows. In Section 2, we present the sufficient conditions used frequently for the positivity of sequences satisfying the recurrence (3). In Section 3, we apply these results to derive the positivity of several combinatorial sequences, including the central Delannoy numbers, the Schröder numbers, and some orthogonal polynomials. Finally in Appendix, we prove Proposition 2.1.

\section{Sufficient conditions for the positivity}

In this section, we give the sufficient conditions for the positivity of $\left\{u_{n}\right\}$ satisfying the recurrence

$$
a_{n} u_{n}=b_{n} u_{n-1}-c_{n} u_{n-2},
$$


where $u_{0}, u_{1} \geqslant 0$ and $a_{n}, b_{n}, c_{n}$ are all nonnegative. Let $x_{n}=\frac{u_{n}}{u_{n-1}}$ for $n \geqslant 1$. In order to establish the positivity of the sequence $\left\{u_{n}\right\}$, it sufficies to check that $\left\{x_{n}\right\}_{n \geqslant 1}$ satisfies

$$
x_{n} \geqslant \frac{c_{n+1}}{b_{n+1}} .
$$

By (3), the sequence $\left\{x_{n}\right\}_{n \geqslant 1}$ satisfies the recurrence

$$
a_{n} x_{n}=b_{n}-\frac{c_{n}}{x_{n-1}} .
$$

Let $p_{n}(x)=a_{n} x^{2}-b_{n} x+c_{n}$ denote the $n$-th characteristic polynomial of the sequence satisfying the recurrence (3). Assume that $b_{n}^{2}-4 a_{n} c_{n} \geqslant 0$ for each $n \geqslant 1$. Then the $n$-th characteristic roots are

$$
\lambda_{n}=\frac{b_{n}-\sqrt{b_{n}^{2}-4 a_{n} c_{n}}}{2 a_{n}} \quad \text { and } \quad \Lambda_{n}=\frac{b_{n}+\sqrt{b_{n}^{2}-4 a_{n} c_{n}}}{2 a_{n}}
$$

respectively. Denote the limit of the sequence $\left\{\lambda_{n}\right\}_{n \geqslant 1}$ by $\lambda_{\infty}$. By a simple calculation and $b_{n}^{2} \geqslant 4 a_{n} c_{n}$, we have

$$
\lambda_{n} \geqslant \frac{c_{n}}{b_{n}}
$$

Hence we can conclude that if $u_{0}, u_{1} \geqslant 0$ and $x_{n} \geqslant \lambda_{n+1}$ for $n \geqslant 1$, then the sequence $\left\{u_{n}\right\}_{n \geqslant 0}$ is nonnegative.

In the following, we suppose that $a_{n}, b_{n}, c_{n}$ are all linear in $n$. More precisely, let

$$
a_{n}=\alpha_{1} n+\alpha_{0}, \quad b_{n}=\beta_{1} n+\beta_{0}, \quad c_{n}=\gamma_{1} n+\gamma_{0}
$$

and denote

$$
A=\left|\begin{array}{cc}
\beta_{0} & \beta_{1} \\
\gamma_{0} & \gamma_{1}
\end{array}\right|, \quad B=\left|\begin{array}{cc}
\gamma_{0} & \gamma_{1} \\
\alpha_{0} & \alpha_{1}
\end{array}\right|, \quad C=\left|\begin{array}{cc}
\alpha_{0} & \alpha_{1} \\
\beta_{0} & \beta_{1}
\end{array}\right| .
$$

We can obtain the monotonicity of the $n$-th characteristic roots $\left\{\lambda_{n}\right\}_{n \geqslant 1}$ and $\left\{\Lambda_{n}\right\}_{n \geqslant 1}$, which is only related to discriminants $A, B, C$.

Proposition 2.1. Suppose that $B^{2} \leqslant A C$. Then the following hold.

(i) If $C \leqslant 0$, then sequences $\left\{\lambda_{n}\right\}_{n \geqslant 1}$ and $\left\{\Lambda_{n}\right\}_{n \geqslant 1}$ are nonincreasing in $n$.

(ii) If $C>0$, then sequences $\left\{\lambda_{n}\right\}_{n \geqslant 1}$ and $\left\{\Lambda_{n}\right\}_{n \geqslant 1}$ are nondecreasing in $n$.

For the sake of the flow, the proof of Proposition 2.1 is given as an Appendix.

We can now give the following sufficient conditions for the positivity of recurrence sequences satisfying (3).

Theorem 2.2. Let $\left\{u_{n}\right\}_{n \geqslant 0}$ be a sequence of integer numbers and satisfy the three-term recurrence (3). Suppose that $C \leqslant 0, B^{2} \leqslant A C$ and $u_{1} \geqslant u_{0} \lambda_{1} \geqslant 0$. Then the positivity problem of the sequence $\left\{u_{n}\right\}_{n \geqslant 0}$ can be solved. 
Proof. Let $x_{n}=\frac{u_{n}}{u_{n-1}}$ for $n \geqslant 1$. We need to prove that $x_{n} \geqslant \lambda_{n+1}$ for all $n \geqslant 1$. From Proposition 2.1 (i), we have $\lambda_{n} \geqslant \lambda_{n+1}$. Hence it suffices to show $x_{n} \geqslant \lambda_{n}$. We proceed by induction on $n$. Clearly, $x_{1} \geqslant \lambda_{1}$ by the condition $u_{1} \geqslant u_{0} \lambda_{1} \geqslant 0$. Now assume that $x_{n-1} \geqslant \lambda_{n-1}$ for $n \geqslant 2$. Note that $p_{n}\left(\lambda_{n}\right)=0$, i.e. $b_{n}-\frac{c_{n}}{\lambda_{n}}=a_{n} \lambda_{n}$. So we have

$$
a_{n} x_{n}=b_{n}-\frac{c_{n}}{x_{n-1}} \geqslant b_{n}-\frac{c_{n}}{\lambda_{n-1}} \geqslant a_{n} \lambda_{n}
$$

by induction hypothesis and Proposition 2.1 (i). Thus $x_{n} \geqslant \lambda_{n}$ for all $n \geqslant 1$. This completes the proof.

Theorem 2.3. Let $\left\{u_{n}\right\}_{n \geqslant 0}$ be a sequence of integer numbers and satisfy the three-term recurrence (3). Suppose that $C>0, B^{2} \leqslant A C, \Lambda_{1} \geqslant \lambda_{\infty}$ and $u_{1} \geqslant u_{0} \Lambda_{1} \geqslant 0$. Then the positivity problem of the sequence $\left\{u_{n}\right\}_{n \geqslant 0}$ can be solved.

Proof. Let $x_{n}=\frac{u_{n}}{u_{n-1}}$. In order to prove the positivity of $\left\{u_{n}\right\}$, it suffices to check that $x_{n} \geqslant \lambda_{n+1}$ for all $n \geqslant 1$. From Proposition 2.1, we have $\Lambda_{1} \geqslant \lambda_{n+1}$. So we only need to show that $x_{n} \geqslant \Lambda_{1}$. We proceed by induction on $n$. Clearly, $x_{1} \geqslant \Lambda_{1}$ by the condition $u_{1} \geqslant u_{0} \Lambda_{1} \geqslant 0$. Now assume that $x_{n-1} \geqslant \Lambda_{1}$ for $n \geqslant 2$. Note that $\lambda_{n} \leqslant \Lambda_{1} \leqslant \Lambda_{n}$ following from Proposition 2.1 and the condition $\Lambda_{1} \geqslant \lambda_{\infty}$. Hence $p_{n}\left(\Lambda_{1}\right)=a_{n} \Lambda_{1}^{2}-b_{n} \Lambda_{1}-c_{n} \leqslant 0$. Furthermore,

$$
a_{n} x_{n}=b_{n}-\frac{c_{n}}{x_{n-1}} \geqslant b_{n}-\frac{c_{n}}{\Lambda_{1}} \geqslant a_{n} \Lambda_{1}
$$

by the induction hypothesis. Then $x_{n} \geqslant \Lambda_{1}$ for all $n \geqslant 1$. The proof is complete.

When $a_{n}, b_{n}, c_{n}$ are all constants, we have $A=B=C=0$ by the definition. So the sufficiency of Theorem 1.1 (i) is a special case of Theorem 2.2. In particular, if $B^{2}=A C$, then we can obtain the following corollary which is interesting and useful from Proposition 2.1, Theorems 2.2 and 2.3.

Corollary 2.4. Let $\left\{u_{n}\right\}_{n \geqslant 0}$ be a sequence of integer numbers and satisfy the three-term recurrence (3). Suppose that $B^{2}=A C$. Then the following results hold.

(i) If $b_{n} C+2 a_{n} B$ has the same sign as $C$ for all $n \geqslant 1$, then the sequence $\left\{\lambda_{n}\right\}_{n \geqslant 1}$ is constant. In addition, if $u_{1} \geqslant u_{0} \lambda_{1} \geqslant 0$, then the positivity problem of the sequence $\left\{u_{n}\right\}_{n \geqslant 0}$ can be solved.

(ii) If $b_{n} C+2 a_{n} B$ has opposite sign of $C$ for all $n \geqslant 1$, then the sequence $\left\{\Lambda_{n}\right\}_{n \geqslant 1}$ is constant. In addition, if $u_{1} \geqslant u_{0} \Lambda_{1} \geqslant 0$, then the positivity problem of the sequence $\left\{u_{n}\right\}_{n \geqslant 0}$ can be solved.

\section{Applications}

In this section, we apply results obtained in the previous section to derive the positivity of several recurrence sequences in a unified manner. 
Let $\nu>-\frac{1}{2}$ be a parameter. The Gegenbauer polynomials sequence $\left\{C_{n}^{(\nu)}(t)\right\}_{n \geqslant 0}$ satisfies the recurrence relation

$$
n C_{n}^{(\nu)}(t)=2 t(\nu+n-1) C_{n-1}^{(\nu)}(t)-(2 \nu+n-2) C_{n-2}^{(\nu)}(t)
$$

with $C_{0}^{(\nu)}(t)=1$ and $C_{1}^{(\nu)}(t)=2 t \nu$. Then we have the following corollary.

Corollary 3.1. The positivity problem of the Gegenbauer polynomials sequence $\left\{C_{n}^{(\nu)}(t)\right\}$ can be solved for $t \geqslant 1, \nu \geqslant \frac{1}{2}$.

Proof. From the recurrence (5), we have $A=-2 t(\nu-1), B=2(\nu-1), C=-2 t(\nu-1)$. Clearly, $b_{n}^{2}-4 a_{n} c_{n}=4\left[\left(t^{2}-1\right) n^{2}+2(\nu-1)\left(t^{2}-1\right) n+t^{2}(\nu-1)^{2}\right] \geqslant 0$ for $t \geqslant 1$ by direct calculation.

First consider the case $t=1$. We have $B^{2}=A C$ and $b_{n} C+2 a_{n} B=-4(\nu-1)^{2} \leqslant 0$. If $\nu>1$, then $C<0$. By Corollary 2.4 (i), we have $\lambda_{n}=1$ for $n \geqslant 1$. And if $\frac{1}{2} \leqslant \nu \leqslant 1$, then $C \geqslant 0$. By Corollary 2.4 (ii), we have $\Lambda_{n}=1$ for $n \geqslant 1$. Thus the positivity of $\left\{C_{n}^{(\nu)}(t)\right\}_{n \geqslant 0}$ follows from Corollary 2.4.

For $t>1$, we have $B^{2} \leqslant A C$. If $\nu>1$, then $C<0$ and if $\frac{1}{2} \leqslant \nu \leqslant 1$, then $C>0$. Also, $\Lambda_{1}=t \nu+\sqrt{t^{2} \nu^{2}-2 \nu+1}$ and $\lambda_{\infty}=t-\sqrt{t^{2}-1}$. Thus the sequence $\left\{C_{n}^{(\nu)}(t)\right\}_{n \geqslant 0}$ is nonnegative from Theorems 2.2 and 2.3 respectively.

In particular, for $\nu=\frac{1}{2}, C_{n}^{\left(\frac{1}{2}\right)}(t)$ reduces to the Legendre polynomials $P_{n}(t)$ and for $\nu=1$, we have $C_{n}^{(1)}(t)=U_{n}(t)$ is the Chebyshev polynomials of the second kind. So the Legendre polynomials sequence $\left\{P_{n}(t)\right\}_{n \geqslant 0}$ and the Chebyshev polynomials sequence $\left\{U_{n}(t)\right\}_{n \geqslant 0}$ are nonnegative for $t \geqslant 1$.

The derivative sequence of Gegenbauer polynomials $\left\{\frac{d}{d t} C_{n}^{(\nu)}(t)\right\}_{n \geqslant 0}$ satisfies the following recurrence relation

$$
(n-1) \frac{d}{d t} C_{n}^{(\nu)}(t)=2 t(\nu+n-1) \frac{d}{d t} C_{n-1}^{(\nu)}(t)-(2 \nu+n-1) \frac{d}{d t} C_{n-2}^{(\nu)}(t)
$$

with $\frac{d}{d t} C_{n}^{(\nu)}(0)=0, \frac{d}{d t} C_{n}^{(\nu)}(1)=2 \nu$ and $\frac{d}{d t} C_{n}^{(\nu)}(2)=4 \nu(\nu+1) t$. Then we have the following.

Corollary 3.2. The positivity problem of the derivative sequence of Gegenbauer polynomials $\left\{\frac{d}{d t} C_{n}^{(\nu)}(t)\right\}_{n \geqslant 0}$ can be solved for $t \geqslant 1, \nu>0$.

Proof. From the recurrence (6), we have $A=-2 t \nu, B=2 \nu, C=-2 t \nu$. And $b_{n}^{2}-4 a_{n} c_{n}=$ $4\left[\left(t^{2}-1\right) n^{2}+2(\nu-1)\left(t^{2}-1\right) n+t^{2}(\nu-1)^{2}+2 \nu-1\right] \geqslant 0$ for $t \geqslant 1$.

For $t \geqslant 1, \nu>0$, we have $C<0$ and $B^{2} \leqslant A C$. Also, $x_{2}=2 t(\nu+1)$ and $\Lambda_{2}=$ $t(\nu+1)+\sqrt{t^{2}(\nu+1)^{2}-(2 \nu+1)}$. Thus the positivity of the sequence $\left\{\frac{d}{d t} C_{n}^{(\nu)}(t)\right\}_{n \geqslant 0}$ follows from Theorem 2.2.

In what follows we list some more examples of recurrence sequences which are easy seen to satisfy the assumption of Theorem 2.3 or Corollary 2.4. Thus the positivity of these sequences is an immediate consequence of Theorem 2.3 or Corollary 2.4. 
Example 3.3. The central Delannoy number $D(n)$ is the number of lattice paths, king walks, from $(0,0)$ to $(n, n)$ with steps $(1,0),(0,1)$ and $(1,1)$ in the first quadrant. It is known that the central Delannoy numbers satisfy the recurrence

$$
n D(n)=3(2 n-1) D(n-1)-(n-1) D(n-2)
$$

with $D(0)=1, D(1)=3$ and $D(2)=14$ (see [12] for a bijective proof). By the recurrence, we have $A=3, B=-1, C=3$. Also, $\Lambda_{1}=3$ and $\lambda_{\infty}=3-2 \sqrt{2}$. Hence the positivity of $\{D(n)\}_{n \geqslant 0}$ follows from Theorem 2.3.

Example 3.4. The (large) Schröder number $r_{n}$ is the number of king walks, Schröder paths, from $(0,0)$ to $(n, n)$, and never rising above the line $y=x$. The Schröder paths consist of two classes: those with steps on the main diagonal and those without. These two classes are equinumerous, and the number of paths in either class is the little Schröder number $s_{n}$ (half the large Schröder number). It is known that the Schröder numbers of two kinds satisfy the recurrence

$$
(n+2) z_{n+1}=3(2 n+1) z_{n}-(n-1) z_{n-1}
$$

with $s_{0}=s_{1}=r_{0}=1, r_{1}=2, s_{2}=3$ and $r_{2}=6$ (see Foata and Zeilberger [4] and Sulanke [16]). By the recurrence, we have $A=9, B=-3, C=9$. Also, $\Lambda_{2}=3$ and $\lambda_{\infty}=3-2 \sqrt{2}$. Hence the positivity of $\left\{r_{n}\right\}_{n \geqslant 0}$ follows from Theorem 2.3.

Example 3.5. Let $h_{n}$ be the number of the set of all tree-like polyhexes with $n+1$ hexagons (Harary and Read [8]). It is known that $h_{n}$ counts the number of lattice paths, from $(0,0)$ to $(2 n, 0)$ with steps $(1,1),(1,-1)$ and $(2,0)$, never falling below the $x$-axis and with no peaks at odd level. The sequence $\left\{h_{n}\right\}_{n \geqslant 0}$ is Sloane's A002212 and satisfies the recurrence

$$
(n+1) h_{n}=3(2 n-1) h_{n-1}-5(n-2) h_{n-2}
$$

with $h_{0}=h_{1}=1$ and $h_{2}=3$. By the recurrence, we have $A=45, B=-15, C=9$. Also, $\Lambda_{2}=3$ and $\lambda_{\infty}=1$. So $\left\{h_{n}\right\}_{n \geqslant 0}$ is nonnegative by Theorem 2.3.

Example 3.6. Let $w_{n}$ be the number of walks on cubic lattice with $n$ steps, starting and finishing on the $x y$ plane and never going below it (Guy [6]). The sequence $\left\{w_{n}\right\}_{n \geqslant 0}$ is Sloane's A005572 and satisfies the recurrence

$$
(n+2) w_{n}=4(2 n+1) w_{n-1}-12(n-1) w_{n-2}
$$

with $w_{0}=1, w_{1}=4$ and $w_{2}=17$. By the recurrence, we have $A=144, B=-36, C=12$. Also, $\Lambda_{1}=4$ and $\lambda_{\infty}=2$. So $\left\{w_{n}\right\}_{n \geqslant 0}$ is nonnegative by Corollary 2.4 .

\section{Concluding Remarks}

Let $u_{0}, u_{1}, u_{2}, \ldots$ be a sequence of nonnegative numbers. The sequence is called $\log$ convex (resp. log-concave) if for all $k \geqslant 1, u_{k-1} u_{k+1} \geqslant u_{k}^{2}$ (resp. $\left.u_{k-1} u_{k+1} \leqslant u_{k}^{2}\right)$. Clearly, 
a sequence $\left\{u_{k}\right\}_{k \geqslant 0}$ of positive numbers is log-convex (resp. log-concave) if and only if the sequence $\left\{u_{k+1} / u_{k}\right\}_{k \geqslant 0}$ is increasing (resp. decreasing). For the positive sequence satisfying the recurrence (3), we have recently established the following result for the log-convexity and log-concavity (see [11] for instance).

Theorem $4.1([11])$. Let $\left\{u_{n}\right\}_{n \geqslant 0}$ be a sequence of positive numbers and satisfy the threeterm recurrence

$$
\left(\alpha_{1} n+\alpha_{0}\right) u_{n+1}=\left(\beta_{1} n+\beta_{0}\right) u_{n}-\left(\gamma_{1} n+\gamma_{0}\right) u_{n-1}
$$

for $n \geqslant 1$, where $\alpha_{1} n+\alpha_{0}, \beta_{1} n+\beta_{0}, \gamma_{1} n+\gamma_{0}$ are positive for $n \geqslant 1$. Suppose that $A C \geqslant B^{2}$. Then the following results hold.

(i) If $B<0, C>0, u_{0} B+u_{1} C \geqslant 0$ and $u_{1}^{2} \leqslant u_{0} u_{2}$, then the sequence $\left\{u_{n}\right\}$ is log-convex.

(ii) If $B>0, C<0, z_{0} B+z_{1} C \leqslant 0$ and $u_{1}^{2} \geqslant u_{0} u_{2}$, then the sequence $\left\{u_{n}\right\}$ is log-concave.

Using Theorem 4.1, we can get that sequences appeared in this paper are either logconcave or log-convex. For example, the central Delannoy sequence $\{D(n)\}_{n \geqslant 0}$ is $\log$ convex [11] and the sequence $\left\{C_{n}^{(t)}(t)\right\}_{n \geqslant 0}$ is log-concave for $\nu \geqslant 1, t \geqslant 1[3]$.

By the same technique used in the proof of Proposition 2.1, Theorems 2.2 and 2.3, we can also give more sufficient conditions for the positivity of sequences satisfying the recurrence (3) when $B^{2}>A C$. As consequences, we can obtain the positivity problem of the Laguerre polynomials sequence $\left\{L_{n}(t)\right\}_{n \geqslant 0}$ can be solved for $t \leqslant 0$.

\section{$5 \quad$ Appendix. Proof of Proposition 2.1}

The purpose of this Appendix is to prove Proposition 2.1.

Proof. We prove the result only for the case $\lambda_{n}$ of (i) since the case (ii) is similar. In order to prove that $\left\{\lambda_{n}\right\}_{n \geqslant 1}$ is nonincreasing, it suffices to show $\lambda_{n}^{\prime} \leqslant 0$ for $n \geqslant 1$. It is easy to get that the derivative of $\lambda_{n}$ with respect to $n$ is

$$
\begin{aligned}
\lambda_{n}^{\prime} & =\left(\frac{b_{n}-\sqrt{b_{n}^{2}-4 a_{n} c_{n}}}{2 a_{n}}\right)^{\prime} \\
& =\frac{\left(a_{n} b_{n}^{\prime}-a_{n}^{\prime} b_{n}\right)\left(\sqrt{b_{n}^{2}-4 a_{n} c_{n}}-b_{n}\right)+2 a_{n}\left(a_{n} c_{n}^{\prime}-a_{n}^{\prime} c_{n}\right)}{2 a_{n}^{2} \sqrt{b_{n}^{2}-4 a_{n} c_{n}}} \\
& =\frac{\left(\alpha_{0} \beta_{1}-\alpha_{1} \beta_{0}\right)\left(\sqrt{b_{n}^{2}-4 a_{n} c_{n}}-b_{n}\right)+2 a_{n}\left(\alpha_{0} \gamma_{1}-\alpha_{1} \gamma_{0}\right)}{2 a_{n}^{2} \sqrt{b_{n}^{2}-4 a_{n} c_{n}}} \\
& =-\frac{b_{n} C+2 a_{n} B-C \sqrt{b_{n}^{2}-4 a_{n} c_{n}}}{2 a_{n}^{2} \sqrt{b_{n}^{2}-4 a_{n} c_{n}}} .
\end{aligned}
$$


After rationalizing numerator, we have

$$
\begin{aligned}
\lambda_{n}^{\prime} & =-\frac{\left(b_{n} C+2 a_{n} B\right)^{2}-C^{2}\left(b_{n}^{2}-4 a_{n} c_{n}\right)}{2 a_{n}^{2} \sqrt{b_{n}^{2}-4 a_{n} c_{n}}\left(b_{n} C+2 a_{n} B+C \sqrt{b_{n}^{2}-4 a_{n} c_{n}}\right)} \\
& =-\frac{2\left(a_{n} B^{2}+b_{n} B C+c_{n} C^{2}\right)}{a_{n} \sqrt{b_{n}^{2}-4 a_{n} c_{n}}\left(b_{n} C+2 a_{n} B+C \sqrt{b_{n}^{2}-4 a_{n} c_{n}}\right)} .
\end{aligned}
$$

Note that $b_{n} B+c_{n} C=-a_{n} A$ since

$$
a_{n} A+b_{n} B+c_{n} C=\left|\begin{array}{ccc}
a_{n} & \alpha_{1} & \alpha_{0} \\
b_{n} & \beta_{1} & \beta_{0} \\
c_{n} & \gamma_{1} & \gamma_{0}
\end{array}\right|=\left|\begin{array}{ccc}
\alpha_{1} n+\alpha_{0} & \alpha_{1} & \alpha_{0} \\
\beta_{1} n+\beta_{0} & \beta_{1} & \beta_{0} \\
\gamma_{1} n+\gamma_{0} & \gamma_{1} & \gamma_{0}
\end{array}\right|=0 .
$$

Hence

$$
\lambda_{n}^{\prime}=-\frac{2\left(B^{2}-A C\right)}{\sqrt{b_{n}^{2}-4 a_{n} c_{n}}\left(b_{n} C+2 a_{n} B+C \sqrt{b_{n}^{2}-4 a_{n} c_{n}}\right)} .
$$

If $C=0$, then $B=0$ since $B^{2} \leqslant A C=0$. Hence we have $A=0$ by the definition. It follows that $\lambda_{n}^{\prime}=0$ from the recurrence (8).

Suppose now that $C<0$. Then $b_{n} C+2 a_{n} B$ is linear in $n$. Note that it changes sign at most once. Without loss of generality, we assume that it changes from nonnegative to nonpositive. Thus we can get $\lambda_{n}^{\prime} \leqslant 0$ first from (8), and then (9). This completes our proof of Proposition 2.1.

\section{Acknowledgment}

The author thanks Prof. Y. Wang, who first ask her about the positivity problem of recurrence sequences and give helpful suggestions in the preparation of this paper.

The author thanks the anonymous referee for careful reading and valuable suggestions.

\section{References}

[1] R. Askey, Certain rational functions whose power series have positive coefficients. II, SIAM J. Math. Anal. 5 (1974) 53-57.

[2] R. Askey, G. Gasper, Certain rational functions whose power series have positive coefficents, Amer. Math. Monthly 79 (1972) 327-341.

[3] T. Došlić, D. Veljan, Logarithmic behavior of some combinatorial sequences, Discrete Math. 308 (2008) 2182-2212.

[4] D. Foata, D. Zeilberger, A classic proof of a recurrence for a very classical sequence, J. Combin. Theory Ser. A 80 (1997) 380-384.

[5] J. Gillis, B. Reznick, D. Zeilberger, On elementary methods in positivity theory, SIAM J. Math. Anal. 14 (1983) 396-398. 
[6] R.K. Guy, Catwalks, sandsteps and Pascal pyramids, J. Integer Seq. 3 (2000) Article 00.1.6.

[7] V. Halava, T. Harju, M. Hirvensalo, Positivity of second order linear recurrent sequences, Discrete Appl. Math. 154 (2006) 447-451.

[8] F. Harary, R.C. Read, The enumeration of tree-like polyhexes, Proc. Edinb. Math. Soc. (2) 17 (1970) 1-13.

[9] M.E.H. Ismail, M.V. Tamhankar, A combinatorial approach to some positivity problems, SIAM J. Math. Anal. 10 (1979) 478-485.

[10] M. Kauers, Computer algebra and power series with positive coefficients, In Proceedings of FPSAC 2007, electronic.

[11] L.L. Liu, Y. Wang, On the log-convexity of combinatorial sequences, Adv. in Appl. Math. 39 (2007) 453-476.

[12] P. Peart, W.-J. Woan, A bijective proof of the Delannoy recurrence, Congr. Numer. 158 (2002) 29-33.

[13] N.J.A. Sloane, The on-line encyclopedia of integer sequences, http://www.research.att.com/ njas/sequences/.

[14] R.P. Stanley, Positivity problems and conjectures in algebraic combinatorics, Mathematics: Frontiers and Perspectives, American Mathematical Society, Providence, RI, 2000, pp. 295-319.

[15] A. Straub, Positivity of Szegö's rational function, Adv. in Appl. Math. 41 (2008) 255-264.

[16] R.A. Sulanke, Bijective recurrences concerning Schröder paths, Electron. J. Combin. 5 (1998), Research Paper 47, 11 pp. 